\title{
Effects of Dislocation Walls on Charge Carrier Transport Properties in CdTe Single Crystal
}

\author{
C. Buis ${ }^{a}$, E. Gros d'Aillon ${ }^{a}$, A. Lohstroh ${ }^{b}$, G. Marrakchi ${ }^{c}$, C. Jeynes ${ }^{b}$, and L. Verger ${ }^{a}$ \\ ${ }^{a}$ CEA, LETI, MINATEC-Campus, 17 rue des Martyrs, F-38054 GRENOBLE. \\ ${ }^{\mathrm{b}}$ Department of physics, University of Surrey, Guilford, GU2 7XH, UK \\ ${ }^{c}$ University of Saint-Etienne, Jean Monnet, F-42000, Saint-Etienne, France \\ Corresponding author: Camille Buis - Present address: CEA, LETI, MINATEC-Campus, 17 rue des Martyrs, F- \\ 38054 GRENOBLE, FRANCE \\ Phone: +33 (0)4-38-78-30-49 \\ e-mail: camille.buis@cea.fr
}

\begin{abstract}
Radiation detectors for medical imaging at room temperature have been developed thanks to the availability of large chlorine-doped cadmium telluride (CdTe:Cl) crystals. Microstructural defects affect the performance of CdTe:Cl radiation detectors. Advanced characterization tools, such as Ion Beam Induced Current (IBIC) measurements and chemical etching on tellurium and cadmium faces were used to evaluate the influence of sub-grain-boundaries on charge carrier transport properties. We performed IBIC imaging to correlate inhomogeneities in charge collection for both types of charge carrier with distribution of dislocation walls in the sample. This information should help improve performance in medical imaging applications.
\end{abstract}

Keywords - CdTe:Cl; IBIC; Chemical etching; Sub-grain-boundary.

This article was published as: Nuclear Instruments and Methods in Physics Research, Section A: Accelerators, Spectrometers, Detectors and Associated Equipment 735:188-192 2014; http://dx.doi.org/10.1016/j.nima.2013.08.084

\section{Introduction}

X-ray imaging sensors with high spatial resolution, high sensitivity and good signal-to-noise ratio, operating at room temperature have been developed over the last decade thanks to the availability of large single crystals of cadmium telluride doped with chlorine $(\mathrm{CdTe}: \mathrm{Cl})$. These crystals measure several $\mathrm{cm}^{2}$ and have good transport properties [1]. CdTe: $\mathrm{Cl}$ has a high absorption coefficient, such that it can absorb photons with high energy. The signal-to-noise ratio for $\mathrm{CdTe}: \mathrm{Cl}$ detectors can be optimized due to the relatively low energy required for electron-hole pair-production [2]-[3]. CdTe: $\mathrm{Cl}$ crystals also have good charge carrier transport properties with an adequate mobility-lifetime product for electrons [4] to make charge collection at the anode efficient. However, defects in single crystals, such as inclusions and dislocations can induce charge transport inhomogeneities which affect detector performance [2]. To study this, we produced a CdTe-based X-ray detector operating in integration mode. We previously showed good correlation between the line features observed in dark-current and X-rayinduced photo-current measurements and dislocation walls revealed by the presence of etch pits on the detector's surface [5]. These etch pits were revealed after Everson etching (after removal of the electrodes). These nonuniformities in the detector response indicate that charge carrier transport is heterogeneous. Previous publications from other groups [6-7] also demonstrate a good correlation between the X-ray response map for a detector, working in counting mode, and its topography, particularly with regard to sub-grain boundaries in the crystal.

Better understanding of how sub-grain boundaries affect charge carrier transport properties should help improve detector performance. In this paper, etching with Everson solution was used to reveal the dislocation density on the tellurium face of the crystal, while Nakagawa solution was used for the cadmium face. Infrared 
(IR) microscopy was used to visualize how tellurium (Te) inclusions are distributed. Ion Beam Induced Current measurements were carried out on the same sample to map the transport properties of both charge carriers.

This article presents results from several characterization methods, brought together and correlated to understand how dislocation walls affect charge carrier transport properties. We believe this is the first time a good correlation between the distribution of etch pits and contrasted features revealed by IBIC measurements have been described for CdTe single crystals.

\section{Experimental setup}

\subsection{IBIC measurements}

The detector-grade material was manufactured from a $16 \mathrm{~mm} \times 16 \mathrm{~mm} \times 1 \mathrm{~mm}$ CdTe single crystal, grown using the travelling heater method (THM) by Acrorad. The crystal is doped with chlorine to achieve high resistivity: 2$3 \times 10^{9} \Omega . c m$ [1]. Single rectangular contacts were deposited on each face with an electroless gold technique without guard ring. Prior to depositing gold, chemical etching with bromine in methanol was performed to remove oxidation from the detector's surface. The sample was then placed in an $\mathrm{AuCl}_{3}$ solution. After gold deposition, the four faces of the sample were mechanically polished to remove the gold layer deposited outside the contacts. The device was mounted on a ceramic substrate, fixed with a conducting adhesive tape. The bias voltage was applied to the top electrode through a gold wire connecting it to an Ortec 142 A preamplifier. The bottom of the device was connected to the earth.

IBIC measurements were carried out at the nuclear micro-beam facility at the University of Surrey. Detector response was measured under irradiation with $4 \mathrm{MeV}$ protons. The protons deposit their energy near the surface of the detector. The resulting charge carriers drift through the bulk of the detector resulting in an induced charge on the collection electrode, which is on the face opposite that being irradiated. The experimental system is described in detail by Sellin et al. [4,8]. The charge sensitive pre-amplifier was an Ortec 142. Following this, the electronic chain splits. One signal is passed through an Ortec 572 shaping amplifier, whose output is passed onto the Omdaq ADC (i.e. resulting in a spectrum in channels). The second pathway is through a Fast filter Amplifier (differentiation and integration both switched off, gain 15) and from there into the digitiser (Ortec 579) [9]. The shaping time setting (Ortec 572, Omdaq channel) was 3 microseconds.

For each type of charge carrier, the variation of the mobility-lifetime product $(\mu \tau)$ was studied based on the Charge Induction Efficiency (CIE). The CIE is expressed as the ratio between the charge induced on the electrodes and the initial charge deposited $\left(\mathrm{Q}_{0}\right)$ by the interaction between protons and the sensor material. In the detector-grade crystal, the penetration depth of a $4 \mathrm{MeV}$-proton is $100 \pm 5 \mu \mathrm{m}$ (as determined using SRIM [10]). This is shallow compared to the total device thickness. We can therefore consider the signal induced by ionizing irradiation to be dominated by the drift of a single type of charge carrier [4]. For cathode irradiation, the charge is induced by electron drift. The CIE can be calculated using the simplified Hecht equation (1), where $\mu_{n}$ and $\tau_{n}$ are the mobility and the lifetime of an electron, $V$ is the applied bias voltage, and $d$ is the sample thickness

$$
C I E(V)=\mu_{n} \cdot \tau_{n} \cdot \frac{V}{d^{2}} \times\left(1-e^{-\left(\frac{d^{2}}{\mu_{n} \cdot V \cdot \tau_{n}}\right)}\right) \text { (1) [4] }
$$

The I-V curve of our sample showed asymmetric contact, with a smaller resistivity on one side of the detector. The sample was therefore removed from the conductive tape and turned over between the acquisition measuring hole induction efficiency and that determining the electron CIE. This operation makes it possible to measure the same dark-current during both acquisitions.

\subsection{Characterization of crystal defects}

The distribution of dislocations on the Te surface was studied by etching with Everson solution (6 mL HF, 24 $\mathrm{mL} \mathrm{HNO}_{3}, 150 \mathrm{~mL}$ lactic acid). Etching time was $150 \mathrm{~s}$ at room temperature. This solution affects the $(\overline{1} \overline{1} \overline{1}) \mathrm{Te}$ face of CdTe by forming regular triangular pyramidal etch pits [11]. Each etch pit corresponds to the exit point for a single dislocation.

How dislocations were distributed on the cadmium surface was investigated by etching with Nakagawa solution, $\left(20 \mathrm{~mL} \mathrm{H}_{2} \mathrm{O}, 20 \mathrm{~mL} \mathrm{H}_{2} \mathrm{O}_{2}, 30 \mathrm{~mL} \mathrm{HF}\right)$. Etching time was $60 \mathrm{~s}$ at room temperature. This solution forms etch pits on the (111)Cd face of CdTe crystal [12]. [13].

Te inclusions were then explored using IR transmission (with an Olympus BX61 Motorized Microscope) 


\section{Results and discussion}

\subsection{IBIC measurements}

CIE was measured for both electron and hole collection at bias voltages of $-5,-6,-8,-10,-11,-15,-19$ and $-23 \mathrm{~V}$ for electrons, and at 25, 28, 32, 38, 76, 95 and $114 \mathrm{~V}$ for holes. An IBIC image was also taken at each bias voltage. These images were used to estimate conditions providing a better contrast when acquiring an IBIC map.

Electron drift through the bulk of the sample generates the map shown in Fig. 1. The total area of the map is $6 \times 3.75 \mathrm{~mm}^{2}$, measured over $8 \times 5$ fields of view (FOV). Each field of view is 750 microns wide and is composed of $256 \times 256$ pixels, so that the spatial resolution is $3 \mu \mathrm{m}$. The map was acquired with an applied voltage of $-19 \mathrm{~V}$. The IBIC images show non-uniform response. Three different kinds of defects are distinguished as follows:

1) Lines presenting lower CIE values (between 2200 and 2400 arbitrary units) than the values recorded for the defect-free-area (between 2300 and 2500 arb. units). The average width of these linear defects is $46 \mu \mathrm{m}$. Extended defects of this type cross the whole width of the FOV, while smaller ones appear tangled (e.g. dashed circle in Fig. 1).

2) Black dots with a mean CIE of 2200 arbitrary units. The average apparent diameter of these defects is 66 $\mu \mathrm{m}$.

3) Larger black defects with a mean CIE of less than 1000 arbitrary units (e.g. top right in Fig. 1).

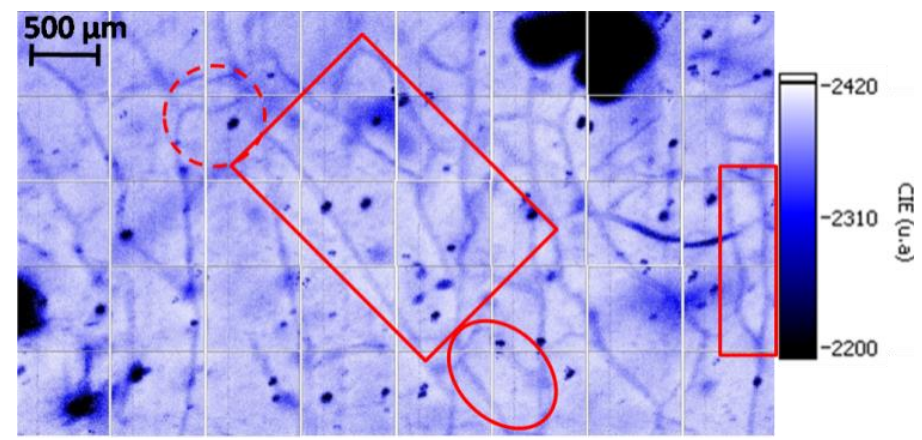

Fig. 1. Electron CIE map acquired at $-19 \mathrm{~V}$. The map corresponds to 40 fields of view, covering an area of $6 \times 3.75 \mathrm{~mm}^{2}$

Fig. 2 shows the map generated by measuring hole drift through the bulk of the sample. The map represents an area of $3.75 \times 3.75 \mathrm{~mm}^{2}$, from 25 fields of view. Data were acquired with an applied voltage of 76 $\mathrm{V}$. The IBIC images show a non-uniform response. The defects observed on this map are similar to those described for electron drift:

1) Lines representing lower CIE values (between 2100 and 2200) compared to the values of the defectfree-area (between 2100 and 2300 arbitrary units). The average thickness of linear defects is $20 \mu \mathrm{m}$.

2) Black dots with a mean CIE of around 1900, with an average apparent diameter of $36 \mu \mathrm{m}$.

3) Larger black defects with a CIE of less than 1020 arbitrary units.

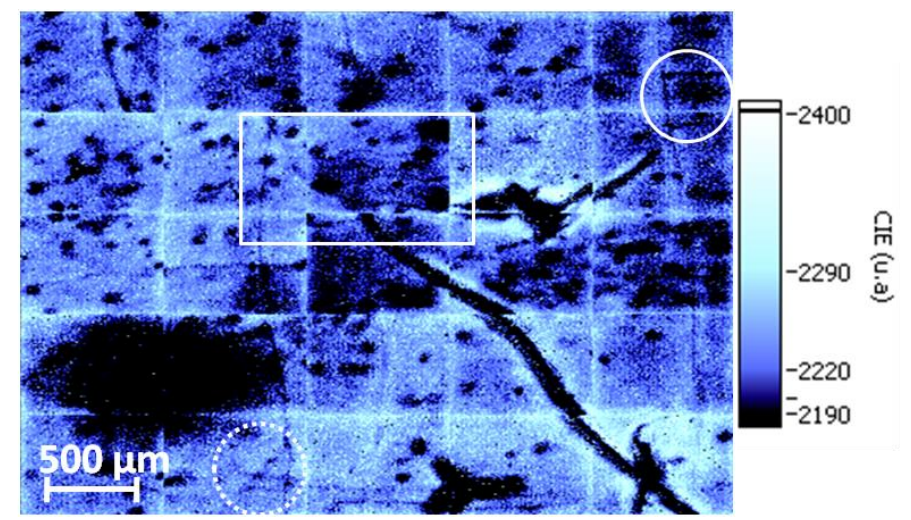

Fig. 2. Hole CIE map acquired at $76 \mathrm{~V}$. The map corresponds to 25 fields of view, covering an area of $3.75 \times 3.75$ $\mathrm{mm}^{2}$. 


\subsection{Characterization of crystal defects}

The electron drift and hole drift patterns for our detector suggested the presence of Te-rich phases. These were investigate by IR microscopy which revealed isolated Te inclusions measuring from less than $5 \mu \mathrm{m}$, for the smallest one, to around $12 \mu \mathrm{m}$, for the biggest one (Fig. 3). These sizes are consistent with previous reports [14]. These inclusions are very uniformly distributed.

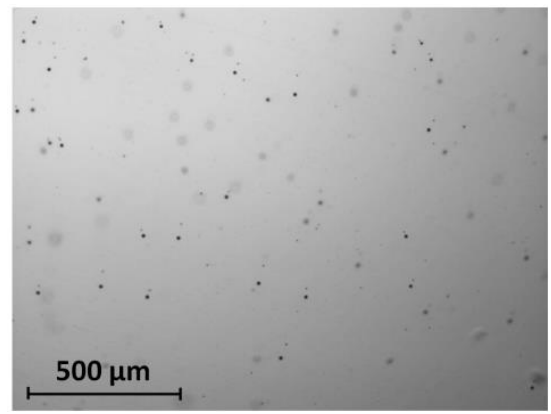

Fig. 3. Visualization of tellurium inclusions by IR transmission microscopy at 5x magnification.

The tellurium and cadmium etched faces are shown in Fig. 4 (a) and (b), respectively. The side length of the etch pits is about $7 \mu \mathrm{m}$ with Everson etching, and $17 \mu \mathrm{m}$ with Nakagawa etching. These images reveal multiple dislocation walls as straight lines nearly all parallel to each other. Other sub-grain-boundaries are tangled. Numerous isolated dislocations are also present (as shown by the multiple small black dots between dislocation walls). Most of the sub-grain boundaries appear to cross the whole thickness of the sample since the distribution of etch pits on both surfaces is similar. This indicates that these boundaries may propagate from the $\mathrm{Te}$ face to the $\mathrm{Cd}$ face in equivalent planes of the glide system for zinc blende crystals $(\langle 110\rangle\{111\})$ [15]. The angle between all equivalent planes in this glide system is $70.53^{\circ}$, which could explain why the network design on the Te face is not exactly the same as the one on the $\mathrm{Cd}$ face.
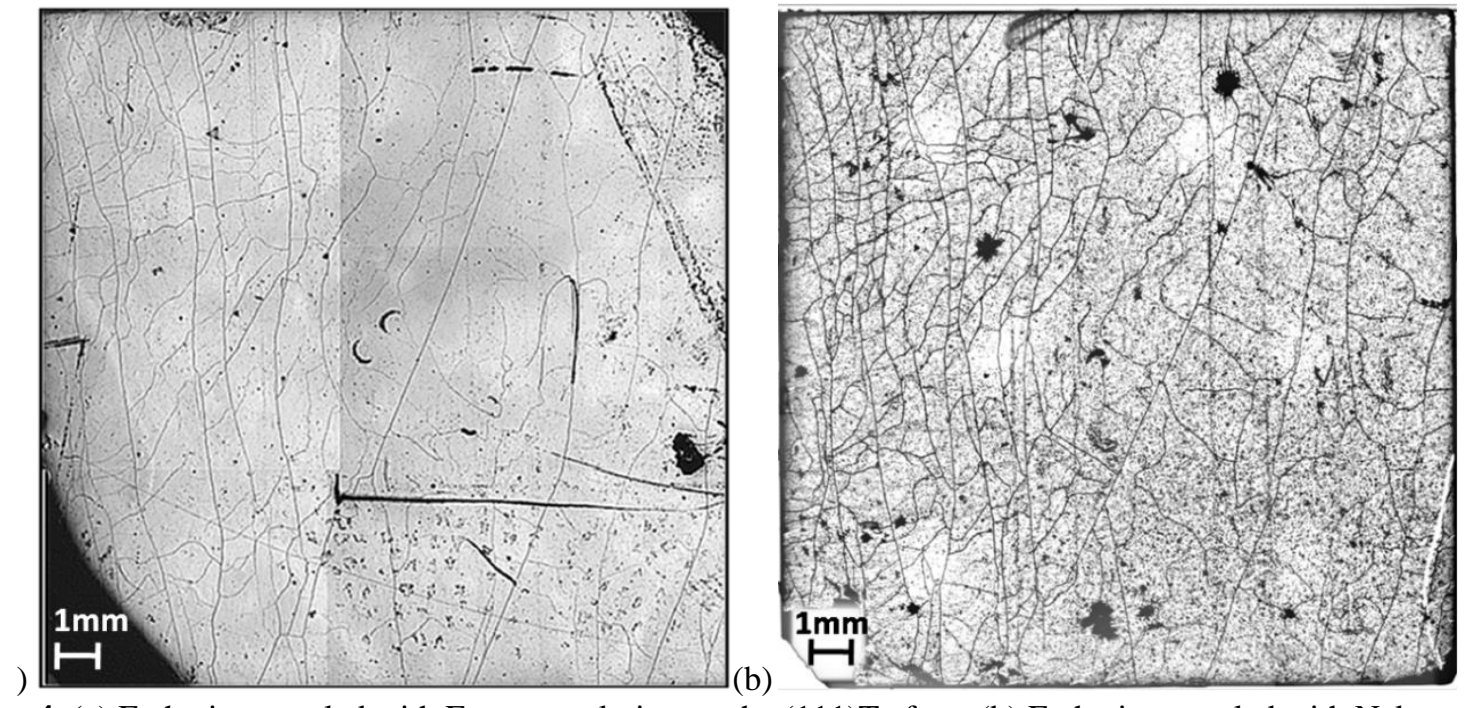

Fig. 4. (a) Etch pits revealed with Everson solution on the (111)Te face. (b) Etch pits revealed with Nakagawa solution on the (111)Cd face.

IBIC measurements were performed in a small part of the sample presented in Fig. 4. The sample was turned over between both IBIC acquisitions, thus the irradiated face is different for the two types of charge carrier: the IBIC map with electron signal (cf. Fig. 1) was taken by irradiating the Te face and the IBIC map with hole signal (cf. Fig. 2) by irradiating the Cd face. The Fig. 5 shows two zooms of Fig. 4 (a) and (b) presenting the etch pits distribution on the scanned area for IBIC measurements. The distribution of etch pits throughout the scanned area for electron induction is shown in Fig. 5 (a), and for hole induction in Fig. 5 (b).

Areas with lower CIE on IBIC maps correlate well with the distribution of the dislocation walls revealed on the sample's surfaces. Actually, the shapes of the line-defects in the electron CIE map (cf. Fig. 1) are similar to the shapes of sub-grain-boundaries on the Te surface presented in Fig. 5 (a) (which is the irradiated area) but their sizes are 5-7 times larger. The rectangles and circles inserted in Fig. 1 and in Fig. 5 (a) highlight zones of 
particular similarity between these two maps. The hole CIE map (cf. Fig. 2) appears to correlate better with the etch pits distribution on the Cd surface in Fig. 5 (b) (which is the irradiated surface). Circles and rectangles on Fig. 2 and Fig. 5 (b) indicate zones of similarity between these two images.

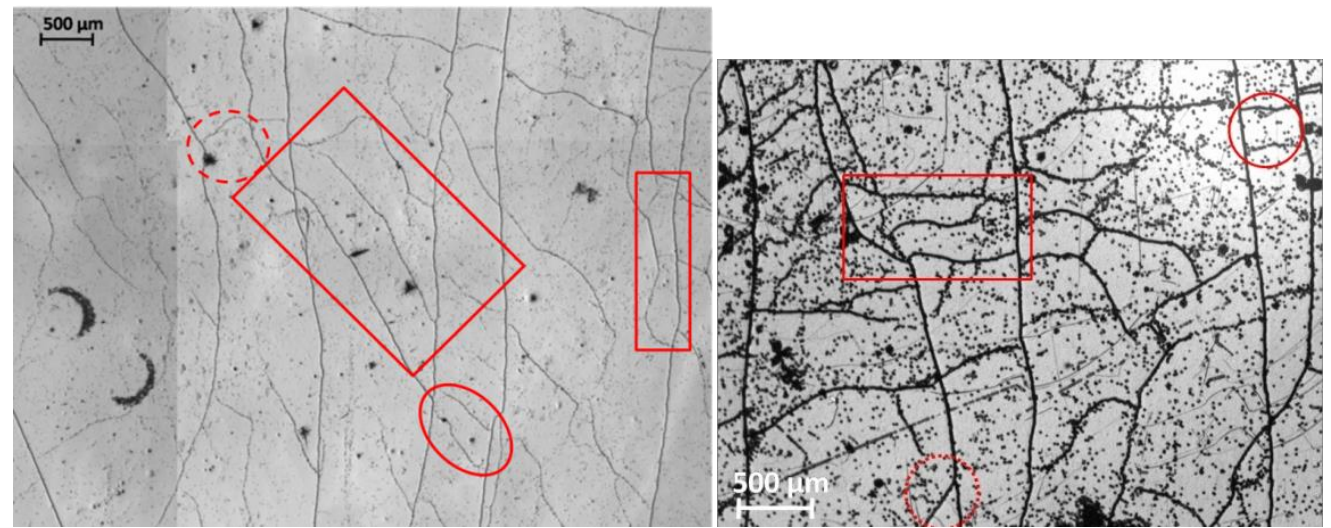

Fig. 5. (a) Etch pits revealed with Everson solution on the (111)Te face of the CdTe:Cl crystal. Image acquired with the microscope in reflection mode at 1,25x magnification. (b) Etch pits revealed with Nakagawa solution on the (111)Cd face of the $\mathrm{CdTe}: \mathrm{Cl}$ crystal. Image acquired with the microscope in reflection mode at $1,25 \mathrm{x}$ magnification.

\subsection{Discussion}

The different techniques to characterize crystal defects indicate that the line-defects with low CIE are dislocation walls which reduce the detector's charge carrier transport properties.

The width of the different defects observed in the IBIC maps could be influenced by the width of the charge carrier cloud created when protons interact with the sensor material. Diffusion of the charge carrier cloud during its drift into the bulk of the sample could also explain why the defects appear larger in IBIC maps (around $50 \mu \mathrm{m}$ ) than on optical microscope images (less than $20 \mu \mathrm{m}$ ). Furthermore the drift and diffusion of charge carrier clouds can also explain why the hole IBIC images are blurred.

We believe that the small dots observed in IBIC maps results from Te inclusions. This second type of defect is uniformly distributed on Fig. 1 and on Fig. 2, with a pattern similar to that observed with IR microscopy (cf. Fig. 3). Furthermore, the size of the black dots (few tens of microns) is consistent with the size of Te inclusions, if we take diffusion of the charge cloud during its drift into account. An exact correlation cannot be proved from the data available here because the surface of the sample was polished before electrode deposition, thus removing inclusions near the surface. The density of Te inclusions revealed by IR transmission microscopy (Fig. 3) is 4-fold that of the black dots on the CIE maps in Fig. 1 and Fig. 2. This is consistent with the diffusion effect: deep-lying inclusions give weak contrast in IBIC because the charge cloud is a lot wider than the defect by the time it reaches it.

The larger defects, with less than 1100 counts for CIE, might be defects deposited on the electrodes.

\section{Conclusion}

For both types of charge carrier, the transport properties of the sample are not homogeneous. This is due to crystal defects, such as dislocation walls and Te inclusions, reducing the mobility-lifetime product of the charge carriers. A very clear correlation was, in fact, established between the distribution of the dislocation network and the linear defects revealed by their lower CIE on the device. Finally, sub-grain boundaries affect performance of CdTe-based radiation detectors and create line-defects with higher photo-current and dark-current [5] by influencing the $\mu \tau$ product of the charge carrier.

\section{ACKNOWLEDGMENTS}

We thank Gilbert Gaude and Marie-Claude Gentet from CEA and Dr Veeramani Perumal from University of Surrey for preparing the sample used in this experiment.

This work was supported by the European Community as an Integrating Activity "Support of Public and Industrial Research Using Ion Beam Technology (SPIRIT)” under EC contract no. 227012. 


\section{REFERENCES}

[1] M. Funaki, T. Ozaki, K. Satoh, and R. Ohno, Growth and characterization of CdTe single crystals for radiation detectors, Nucl. Instrum. Meth. 436 (1999) 120.

[2] C. Szeles, Advances in the crystal growth and device fabrication technology of CdZnTe room temperature radiation detectors, IEEE Trans. Nucl. Sci. 51 (2004) 1242.

[3] M. Hage-Ali, P. Siffert, Semiconductors for Room Temperature Nuclear Detector Applications, vol. 43, edited by T.E. Schlesinger and R.B. James, Academic Press, pp. 291-331, (1995).

[4] P.J. Sellin, A.W. Davies, F. Boroumand, A. Lohstroh, M.E. Özsan, J. Parkin, and M. Veale, IBIC characterization of charge transport in CdTe:Cl, Semiconductors. 41 (2007) 395.

[5] C. Buis, G. Marrakchi, T. A. Lafford, A. Brambilla, L. Verger, and E. Gros d'Aillon, Effects of Dislocation Walls on image quality when using Cadmium Telluride X-Ray Detectors. IEEE Trans. Nucl. Sci. 60 (2013) 199.

[6] A. Cecilia, E. Hamann, C. Haas, D. Greiffenberg, A. Danilewsky, D. Haenscke, A. Fauler, A. Zwerger, G. Buth, P. Vagovic, T. Baumbacha, and M. Fiederle, Investigation of crystallographic and detection properties of CdTe at ANKA synchrotron light source, 2011 JINST 6 P10016.

[7] E. Hamann, A. Cecilia, P. Vagoviĉ, D. Hänschke, Butzer, J. Greiffenberg, D. Fauler, A. Baumbach, and M. Fiederle, Applications of Medipix2 single photon detectors at the ANKA synchrotron facility, IEEE Nuclear Science Symposium Conference Record. (2010) 3860.

[8] P.J. Sellin, A.W. Davies, S. Gkoumas, A. Lohstroh, M.E. Özsan, J. Parkin, V. Perumal, G. Prekas, and M. Veale, Ion beam induced charge imaging of charge transport in CdTe and CdZnTe, Nucl. Instrum. Meth. 266 (2008) 1300.

[9] P.J. Sellin, A. Lohstroh, A. Simon, M.B.H. Breese, Digital IBIC - New spectroscopic modalities for ion-beam-induced charge imaging, Nuclear Instruments and Methods in Physics Research, Section A: Accelerators, Spectrometers, Detectors and Associated Equipment, 521 (2004) 600-607.

[10] J.P. and L.G. Haggmark, A Monte Carlo computer program for the transport of energetic ions in amorphous targets, Nucl. Instrum. Meth. 174 (1980) 257.

[11] W. J. Everson, C. K. Ard, J. L. Sepich, B. E. Dean, G. T. Neugebauer and H. F. Schaake, Etch pit characterization of CdTe and CdZnTe substrates for use in mercury cadmium telluride epitaxy, Journal of Electronic Materials. 24 (1995) 505.

[12] K. Nakagawa, K. Maeda, and S. Takeuchi, Observation of dislocations in cadmium telluride by cathodoluminescence microscopy, Applied Physics Letters. 34 (1979) 574.

[13] N. Zambelli, L. Marchini, M. Zha, and A. Zappettini, Three-dimensional mapping of tellurium inclusions in CdZnTe crystals by means of improved optical microscopy, Journal of Crystal Growth. 318 (2011) 1167.

[14] H. Shiraki, M. Funaki, Y. Ando, A. Tachibana, S. Kominami, and R. Ohno, THM growth and characterization of 100 mm diameter CdTe single crystals, IEEE Trans. Nucl. Sci. 56 (2009) 1717.

[15] D.T.J. Hurle, and P. Rudolph, A brief history of defect formation, segregation, faceting, and twinning in melt-grown semiconductors, Journal of Crystal Growth. 264 (2004) 550. 